\title{
The role of fish scale derived scaffold and platelet rich plasma in healing of rabbit tibial defect: an experimental study
}

\author{
Nikta Mansouri ${ }^{1}$, Hamidreza Fattahian ${ }^{1}$, Kimia Mansouri ${ }^{1}$, Pargol Ghavam Mostafavi², \\ Abdolmohammad Kajbafzadeh ${ }^{3}$ \\ ${ }^{1}$ Islamic Azad University, Science and Research Branch, Faculty of Specialized Veterinary Sciences, \\ Department of Clinical Science, Tehran, Iran \\ ${ }^{2}$ Islamic Azad University, Science and Research Branch, Graduate School of Marine Science and Technology, \\ Department of Marine Biology, Tehran, Iran \\ ${ }^{3}$ Tehran University of Medical Sciences, Pediatrics Center of Excellence, Department of Pediatric Urology, \\ Pediatric Urology Research Center, Tehran, Iran
}

Received October 18, 2017

Accepted December 7, 2018

\begin{abstract}
Fish scale is rich in collagen type I and hydroxyapatite, resembling bone structure. It is readily available, cost effective and can compensate for the limitations of grafting methods such as unavailability, zoonotic disease transmission, and high cost. The aim of this study was to evaluate in vivo the fish scale potential and the possible synergistic effect of platelet rich plasma (PRP) with this scaffold in bone regeneration. Fifteen male white New Zealand rabbits were randomly divided into six groups, each involving 5 limbs. Full thickness bicortical defects were created in the proximal tibia of both pelvic limbs of rabbits. The defect was left untreated in the negative control group. In experimental groups the defect was filled with PRP (group 1), cellular fish scale (group 2), combination of cellular fish scale and PRP (group 3), acellular fish scale (group 4), and a combination of acellular fish scale and PRP (group 5). Fresh fish scales were decellularized to increase biocompatibility and reduce immunity reactions. Decellularization was confirmed by DAPI (4',6-diamidino-2-phenylindole) staining. The microstructure and surface characteristics of fish scales were assessed by scanning electron microscopy (SEM). Histopathological evaluation of bone healing was performed on day 56. Although there was no significant difference in the bone union among experimental groups, the union was superior in all experimental groups compared to control. Spongiosa and cortex formation were superior in the acellular groups. Furthermore, PRP promoted bone marrow formation. We concluded that fish scale is a biocompatible scaffold with a high regenerative potential.
\end{abstract}

Acellular, fish, collagen, platelet rich plasma, bone

Bone is the most important structural component of the vertebrate body (Buckwalter and Cooper 1987; Sarkar and Lee 2015). Structural and functional continuance of such tissue is indebted to the balanced bone formation and resorption (Liao et al. 2011; ZigdonGiladi et al. 2015). Numerous bone diseases such as multiple trauma, osteosarcoma, osteomyelitis and osteoporosis are associated with extensive bone damage; bone grafts and biologic scaffolds are required for repairing such defects (Mc Mah on et al. 2013; Wang et al. 2013; Kovach et al. 2015). Finding an ideal bone substitute with suitable physical and mechanical characteristics has been the main focus of researchers heretofore (ZigdonGiladi et al. 2015). Every year more than 2 million bone grafts are performed, with more than 500 of them in the United States (Liao et al. 2011; Oryan et al. 2014; Campana et al. 2014). Ideal bone grafts should be osteoconductive, osteoinductive and osteogenetic. Bone grafts are classified as autograft, allograft and xenograft (Liao et al. 2011; Dimitriou et al. 2011; Campana et al. 2014). Autograft possesses some advantages, however, low availability, prolonged surgery time, haemorrhage and pain have limited its application. High expense, immunogenicity, characteristic changes during provision and diseases 
such as human immunodeficiency virus (HIV) and hepatitis impede allograft application (Dimitriou et al. 2011; Campana et al. 2014; Sarkar and Lee 2015). Xenograft is more available and cost effective than the two aforementioned options (Oryan et al. 2014). Researchers have so far used xenogenic sources such as bovine, ovine, porcine tissues with different preparation methods including demineralization, deproteinization and lyophilization (Dong et al. 2012; Sawkins et al. 2013; Oryan et al. 2014). Xenografts containing collagen and hydroxyapatite increase osteoblast differentiation and enhance osteogenesis, and previous studies have also suggested their biocompatibility (Navarro et al. 2008; Oryan et al. 2014). Risk of immunogenicity, zoonotic diseases and some religious or cultural restrictions have reduced the use of bovine, ovine, and porcine tissues and induced motivation to find other collagen containing biomaterials such as marine sources (Pati et al. 2012; Yamamoto et al 2014; Silva et al. 2014). A remarkable proportion of fish $(75 \%)$ accounts as waste, including skin, bone, fin, the digestive system and scales from which collagen is extractable (Silva et al. 2014). Fish scale matrix mostly consists of collagen and hydroxyapatite which are the main components of bone. This similarity makes it a useful bone substitute (Chou et al. 2014; Silva et al. 2014). Availability, abundance and cost-effectiveness are some other advantages of fish scales (Oryan et al. 2014; Silva et al. 2014). Different decellularization methods such as hydrostatic pressure, enzymatic, physical, and chemical processes have been used to reduce reputed disadvantages of xenografts (Hashimoto et al. 2011; Dong et al. 2012; Oryan et al. 2014; To Minh et al. 2014). During decellularization the cellular structure of tissue is removed, however, the complex composition of structural and functional proteins is preserved (Gilbert et al. 2006; To Minh et al. 2014). Recently scientists used orthobiologics such as platelet rich plasma (PRP) to accelerate bone repair. Effectiveness of PRP in bone healing, lowering infection, haemorrhage and pain management has been reported in several articles. However, PRP effectiveness is still controversial and is the subject of interest in bone research (Alsousou et al. 2009; Civinini et al. 2011; Roffi et al. 2013; Campana et al. 2014). The aim of this study was to assess acellular fish scale effect in the healing of rabbit tibial bone defect and evaluate the potential synergistic effect of PRP with this scaffold.

\section{Materials and Methods}

Preparation of fish scales

Fresh grass carp were obtained from a commercial retailer in Iran, stored at $4{ }^{\circ} \mathrm{C}$ and immediately transported to the laboratory. Fresh scales were harvested and the adherent tissue and fat were subsequently cleaned with distilled water. The scales were transferred to a salt saturated solution of hydrochloric acid and stored in antibiotic containing phosphate buffer saline (PBS) prior to decellularization.

\section{Decellularization of fish scales}

Demineralized fish scales were rinsed in distilled water for $24 \mathrm{~h}$. Samples were decellularized in sodium dodecyl sulphate (SDS), rinsed with distilled water and chemically sterilized in ethanol. Acellular samples were transferred to sterile PBS and stored in $-20^{\circ} \mathrm{C}$.

\section{Characterization of decellularized fish scales}

Decellularization efficiency was assessed using 4',6-diamidino-2-phenylindole (DAPI) cell nucleus staining and scanning electron microscopy (SEM). Samples of decalcified cellular scales and decalcified acellular scales were mounted on slides. To obtain 1000X DAPI stock, $0.2 \mathrm{mg}$ DAPI was dissolved in one ml distilled water and the resultant solution was stored at $4{ }^{\circ} \mathrm{C}$ in dark. To prepare McIlvaine buffer of $\mathrm{pH} 7.0$, two parts of stock solution $\mathrm{A}(0.1 \mathrm{M}$ citric acid $)$ and 8 parts of stock solution $\mathrm{B}\left(0.2 \mathrm{M} \mathrm{Na}_{2} \mathrm{HPO}_{4}\right)$ were mixed. Finally the samples were stained with a combination of $10 \mathrm{ml}$ McIlvaine buffer of $\mathrm{pH} 7.0$ and $10 \mathrm{ml} 1000$ X DAPI stock. Stained slides were examined using a fluorescence microscope at an excitation wavelength of $350 \mathrm{~nm}$. Scanning electron microscope (SERON AIS-2100, Amirkabir University of technology, Tehran, Iran) was used to examine the surface morphology of the cellular and acellular scales after sputtering with gold ions.

In vivo biocompatibility, fluoroscopy and histopathological analysis

All animal experiments were performed according to the guidelines of the Iran Society for the Prevention of Cruelty to Animals (SPCA) and were approved by the Iranian Ethical Framework for Animal Research under the 
reference number IAEC 4-08/1. Fifteen male white New Zealand rabbits of similar weight and age were kept in individual cages for 14 days prior to the experiments to allow adaptation to the lab environment. In order to reduce the sample size and involve fewer animals, we included both legs of each rabbit in separate groups. Animals were randomly divided into 6 groups, each involving 5 limbs (Table 1). The defined groups were negative control (left legs of 5 rabbits) with the defect left untreated; group 1 (right legs of the same 5 rabbits included in the negative control group) with the defect filled with only PRP; group 2 (left legs of different 5 rabbits) with the defect filled with raw scale; group 3 (right legs of the same rabbits as in group 2) with raw scale and PRP used as defect filler; group 4 (left legs of another five rabbits) with acellular scale used as defect filler; and group 5 (right legs of the same rabbits as in group 4) with acellular scale and PRP used as defect filler. It should be noted that PRP was added to the right side defect within the groups. Anaesthesia was induced by an intramuscular combination of $40 \mathrm{mg} / \mathrm{kg}$ ketamine hydrochloride (Ketamine, Alfasan, Woerden, Netherlands) and $0.2 \mathrm{mg} / \mathrm{kg}$ medetomidine hydrochloride (Dorbene Vet ${ }^{\mathbb{B}}$, Spain SYVA s.a.u, Spain) and maintained by 3\% isoflurane (AErrane, Baxter Healthcare Corp, USA). After the clipping and scrubbing of both legs from the mid-shaft femur to the distal tibia, animals were positioned in dorsal recumbency and sterile draping was done. Skin was incised on the dorsomedial region of the crus (Plate VI, Fig. 1A). After subcutaneous dissection and periosteal elevation (Fig. 1B) a full thickness bicortical defect was made in the bone using an orthopaedic drill (number 3.5) (Figs 1C,D). In the negative control group, the defect was left untreated. In group 1 the defect was filled with autologous PRP (Fig. 1E) and then periosteal and skin incision was closed (Fig. 1F). In groups 2-4, the defects were filled with the cellular scale, cellular scale and PRP, acellular scale, and acellular scale and PRP, respectively. Skin and subcutaneous incisions were closed routinely (Fig. 1F). Ten $\mathrm{mg} / \mathrm{kg}$ enrofloxacin (Razak Laboratory, Tehran, Iran) and $0.3 \mathrm{mg} / \mathrm{kg}$ meloxicam (Razak Laboratory, Tehran, Iran) were administered post-operatively for 3 days. The animals were transferred to well-padded cages for $24 \mathrm{~h}$. It should be noted that no anorexia or discomfort were observed despite the involvement of both legs. All animals were able to walk properly. Similar nutritional and environmental conditions were maintained for 8 weeks. Fluoroscopy of the surgical sites was done immediately after surgery and before euthanasia on day 56 to evaluate bone integrity and proper installation of implants (Plate VI, Fig. 2). Specimens were fixed in $10 \%$ neutral buffer formalin solution, decalcified in a hydrochloric and formic acid combination and embedded in paraffin. Haematoxyline and eosin (H\&E) stained slides were prepared from successive sections of paraffin blocks.

Table 1. Defined experimental groups

\begin{tabular}{|c|c|c|c|c|c|c|}
\hline & $\begin{array}{l}\text { Negative control } \\
\text { group }\end{array}$ & $\begin{array}{l}\text { Experimental } \\
\text { group } 1\end{array}$ & $\begin{array}{l}\text { Experimental } \\
\text { group } 2\end{array}$ & $\begin{array}{l}\text { Experimental } \\
\text { group } 3\end{array}$ & $\begin{array}{l}\text { Experimental } \\
\text { group } 4\end{array}$ & $\begin{array}{c}\text { Experimental } \\
\text { group } 5\end{array}$ \\
\hline \multicolumn{7}{|l|}{ No treatment } \\
\hline \multicolumn{2}{|c|}{ Platelet rich plasma } & $\checkmark$ & & $\checkmark$ & & $\checkmark$ \\
\hline \multicolumn{2}{|c|}{ Cellular scale } & & $\checkmark$ & $\checkmark$ & & \\
\hline \multicolumn{2}{|l|}{ Acellular scale } & & & & $\checkmark$ & $\checkmark$ \\
\hline
\end{tabular}

\section{Platelet rich plasma preparation}

After the clipping of the left thoracic region adjacent to the heart, the animals were anaesthetized with intramuscular injection of $40 \mathrm{mg} / \mathrm{kg}$ ketamine hydrochloride (Ketamine, Alfasan, Woerden, Netherlands) and $0.2 \mathrm{mg} / \mathrm{kg}$ medetomidine hydrochloride (Dorben vet ${ }^{\circledR}$, Spain SYVA s.a.u, Spain) and positioned in lateral recumbency. Eight $\mathrm{ml}$ of fresh blood were obtained intracardially and immediately stored into acid citrate dextrose anticoagulant vacutainer tubes. Subcutaneous Ringer solution (Alborz daru, Tehran, Iran) was administered to all animals. Total blood cell count was measured (veterinary cell counter, Nihon Kohden, Japan) and collected blood was centrifuged (Sigma, Germany) at $580 \times g$ for $20 \mathrm{~min}$, subsequently single plasma fraction was collected and further centrifuged at $1,304 \times g$ for $15 \mathrm{~min}$. The $0.5 \mathrm{ml}$ bottom content of tube containing precipitated platelets were collected and platelet count was performed on the samples; when it was $3-5 \times$ higher than the baseline platelet counts, the samples were accepted as PRP.

\section{Results}

\section{Morphological characteristics of fish scales}

The DAPI staining revealed homogenously dispersed cells with blue nuclei in cellular fish scale (Plate VI, Fig. 3). Furthermore, DAPI confirmed a decellularization process and no nuclei were identified in decellularized scales (Fig. 3). Collagen fibres were detectable in both cellular and acellular scales without major structural changes. According to SEM 
Table 2. Histopathological results based on bone healing scoring indices (union, spongiosa, cortex, bone marrow).

\begin{tabular}{|c|c|c|c|c|c|c|c|c|}
\hline \multirow[b]{2}{*}{ Index } & \multirow[b]{2}{*}{ Evaluation scale } & \multirow[b]{2}{*}{ score } & \multicolumn{5}{|c|}{ Experimental Groups } & \multirow[b]{2}{*}{ Group 5} \\
\hline & & & $\begin{array}{c}\text { Negative } \\
\text { control }\end{array}$ & Group 1 & Group 2 & Group 3 & Group 4 & \\
\hline \multirow{5}{*}{ Union } & No sign of union & 0 & & & & & & \\
\hline & Fibrous union & 1 & & & & & & \\
\hline & Osteochondral union & 2 & 1 & 2 & 3 & 3 & 3 & 3 \\
\hline & Bone union & 3 & & & & & & \\
\hline & Complete reorganization & 4 & & & & & & \\
\hline \multirow{5}{*}{ Spongiosa } & No sign of cellular activity & 0 & & & & & & \\
\hline & Early bone formation & 1 & & & & & & \\
\hline & Active new bone formation & 2 & 2 & 3 & 3 & 4 & 4 & 4 \\
\hline & Reorganized spongiosa formation & 3 & & & & & & \\
\hline & Complete reorganized formation & 4 & & & & & & \\
\hline \multirow{5}{*}{ Cortex } & Absence of cortex & 0 & & & & & & \\
\hline & Early detection & 1 & & & & & & \\
\hline & Initiation of formation & 2 & 2 & 3 & 3 & 3 & 4 & 4 \\
\hline & Reorganization in majority & 3 & & & & & & \\
\hline & Complete organization & 4 & & & & & & \\
\hline \multirow{6}{*}{$\begin{array}{l}\text { Bone } \\
\text { marrow }\end{array}$} & Not available & 0 & & & & & & \\
\hline & Detection of fibrinous material & 1 & & & & & & \\
\hline & Defect occupying more than half & 2 & 2 & 3 & 3 & 4 & 3 & 4 \\
\hline & Fully occupying the red bone & & & & & & & \\
\hline & marrow & 3 & & & & & & \\
\hline & Adult type fatty marrow & 4 & & & & & & \\
\hline Summation & & 16 & 7 & 11 & 12 & 14 & 14 & 15 \\
\hline
\end{tabular}

results, fairly squared well-organized tight layers of collagen fibres were viewed in both processed and un-processed scales. It should be noted that collagen fibres were viewed distracted and teared in some zones due to prior SEM processing (Plate VII, Fig. 4).

Histopathological and fluoroscopic analysis

Prepared H\&E stained slides were examined by light microscopy. Union, spongiosa, cortex and bone marrow indices were defined based on Korkmaz et al. (2005) (Table 2 ) and each group was scored for each index. Bone union was superior in groups $2-5$ in comparison to the negative control group and group 1. Reorganized spongiosa formation and complete reorganized formation was seen in all groups except negative control and group 1 after two months' follow up. Cortex index was superior in groups 4 and 5 in comparison to other groups. Bone marrow development was more favourable in groups 3 and 5. In the negative control group a prominent amount of fibrous tissue was identified at the defect site, and also a cartilaginous callus was present to some extent. Furthermore, bone marrow cavities and scant cancellous tissue were observed (Plate VIII, Fig. 5). In group 1, osteocartilaginous callus, chondrocytes, osteocytes and haematopoietic bone marrow were detected (Plate VIII, Fig. 6). In groups 2 and 3, osseous tissue along with hypertrophic osteoblasts were indicative of good healing and osteoclasts were noticed around the scale residues (Plate IX, Figs 7 and 8). Severe inflammatory 
response surrounding fish scale residues was noted in group 3 (Fig. 8). In groups 4 and 5, a significant amount of newly formed osseous tissue containing hypertrophic osteocytes was observed and presence of haematopoietic bone marrow was seen in the newly formed callus; furthermore, osteoclasts were accumulated around acellular scale remnants and an inflammatory response was present (Plate X, Figs 9 and 10). In the histopathological analysis, fish scale generally showed good biocompatibility. According to fluoroscopic images on days 1 and 56 (Fig. 2), no fracture or impaired integrity was seen in any groups and all implants were placed properly in the defect.

\section{Discussion}

Heretofore different bone graft methods have been used to advance the healing of large bone defects. Among various kinds of bone grafts, xenografts have attracted researchers' attention in recent years due to the limitations of other methods such as low availability and expensiveness (Campana et al. 2014; Zigdon-Giladi et al. 2015). Develioglu et al. (2009) reported a positive effect of xenograft in the healing of a rat skull defect. Bansal et al. (2009) yielded promising results in the treatment of a tibial plateau fracture with a bovine cancellous bone xenograft. In this study we used decellularized fish scale as a new xenogenic biomaterial.

Collagen as an essential component of the extracellular bone matrix and a connective tissue support is the most utilized biomaterial which is achievable from different sources (Chou et al 2014; Yamada et al. 2014). In previous studies, mammals such as cattle and swine were the most predominant source of collagen but in recent years the use of such sources is being disputed on grounds of religious reasons and life threatening zoonotic diseases such as bovine spongiform encephalitis, cysticercosis, and tuberculosis. Marine collagen has therefore been introduced as a new source, with scientists all over the world starting to assess its role in tissue healing (Campana et al. 2014; Silva et al. 2014). According to Pati et al. (2012) and Silva et al. (2014), collagen of marine origin is a unique biocompatible and biodegradable source that stimulates cell growth. In addition, it bears a high stretch strength and has low immunogenicity; therefore, it has opened new possibilities in tissue healing. A freeze-dried composite of chitosan, hydroxyapatite and collagen of the sponge Ircinia fusca revealed good attachment and proliferation of MG-63 cells (Silva et al. 2014). Pati et al. (2012) utilized fresh water fish collagen for preparing biocompatible scaffolds. In a similar study, Hoyer et al. (2012) reported acceptable proliferation of human mesenchymal stem cells on a composite of mineralized salmon skin collagen and hydroxyapatite. Yamada et al. (2014) showed a positive effect of fish collagen peptides on osteoblastic cell line culture. In a study by Matsumoto et al. (2015), tilapia collagen fibrils were reported to be effective in differentiation of human mesenchymal stem cell-derived osteoblasts.

Although many studies evaluated marine biomaterials in vitro, in vivo characteristics of these sources have not been completely studied. Fish scale is available at low cost; it is considered as a waste in our country and has so far remained without use. Therefore, here we assessed the potential of cellular and acellular scales of grass carp in vivo in a tibial bone defect of rabbits, achieving promising results based on the histopathological results (Table 2). A number of crosslinking methods have been used in order to enhance the mechanical characteristics of collagen. However, this method induced changes in the implant degradation speed (Chou et al. 2014). In addition, toxicity induced by residues of chemical agents such as glutaraldehyde and carbamide has been proven whilst physical methods do not show toxicity meanwhile establishing adequate physical strength (Chou et al. 2014; Yamada et al. 2014). In this study 
we preferred to assess the effect of fish scale on bone repair without any subsequent physical or chemical strengthening method. Immunogenic reactions of xenografts confirm the necessity of decellularization in the use of a xenogenic source.

In order to remove the cell cytoplasmic and nuclear antigens while preserving the mechanical and functional characteristics, various enzymatic, chemical and physical decellularization methods have been used (Gilbert et al. 2006; Oryan et al. 2014). For instance, Krishnan et al. (2012) compared the physicochemical and mechanical characteristics of acellular fish scale with the human amniotic membrane for corneal tissue engineering and reported encouraging results for the fish scale. In a similar study, Yuan et al. (2014) used biocornea derived from fish scale and reported high biocompatibility after a long-term evaluation. In the present study, grass carp fish scales were decellularized by SDS and ethanol; decellularization was confirmed with DAPI staining and SEM analysis (Plate VI, Figs 1 and 2).

Processed matrices should become sterilized before experimental use. According to a study by $\mathrm{Chou}$ et al. (2014), gamma irradiation induces less injury to collagen fibres in comparison to autoclave meanwhile it has a low effect on elasticity and osteoconductivity of the scaffold. Therefore, in this study we sterilized unprocessed fish scales by gamma irradiation and acellular fish scales became sterilized during decellularization stages in ethanol. No further sterilizing process was needed.

Thamamongood et al. (2012) showed similarity in genetic pathways of intramembranous bone repair and gold fish scale regeneration and introduced it as a promising source in bone healing. In this study we evaluated fish scale as a bone substitute for a proximal tibial defect in the rabbit and our study confirmed fish scale as a biocompatible biomaterial in bone healing based on the histopathological results (Table 2). Chou et al. (2014) reported expressive results after fixation of rabbit femoral fracture with acellular fish scale-derived bone pins.

Growth factors found in healthy bone such as the vascular endothelial growth factor, platelet-derived growth factor and bone morphogenetic proteins may play a role in bone repair (Oryan et al. 2014). It was reported that growth factors can promote proliferation and differentiation of mesenchymal stem cells and increase synthesis and migration of collagen and extracellular matrix. They may also stimulate blood vessels formation (Hakimi et al. 2010; Civinini et al. 2011). Since PRP is an easy obtaining cost-effective source of growth factors, it has been used in combination with grafts and other natural and synthetic scaffolds such as hydroxyapatite, coral skeleton, chitosan, polymers and hydrogels (Roffi et al. 2013; Oryan et al. 2014; Salamanna et al. 2015). Durmuslar et al. (2014) assessed bovine hydroxyapatite, PRP and resorbable collagen membranes for the treatment of intra-bony defects and reported healing acceleration. In another study, Schneppendahl et al. (2015) evaluated an autograft and PRP in a diaphyseal bone defect and obtained desired histomorphometric and radiographic results. Furthermore, Yilmaz et al. (2011) evaluated the healing of intrabony defects after treatment with bovine-derived xenograft combined with platelet-rich plasma versus platelet-poor plasma and similar outcomes were yielded from these two. Since literature review revealed controversial results regarding the role of PRP in bone repair, and the effect of a fish scale xenograft and PRP was not investigated, the authors decided to assess the possible synergistic effect of PRP with fish scale on bone healing in rabbit.

In this study, we used SDS and ethanol in order to decellularize grass carp scale, compromise immune reactions and enhance biocompatibility of fish scale as a xenograft. The decellularization process was confirmed by DAPI cell nucleus staining and SEM analysis. To evaluate biocompatibility, the harvested scaffold was implanted in the rabbit tibial bone defect and according to indices defined in the histological study, 
although unprocessed fish scale had an admissible effect on the healing of bone defect, it was inferior relative to acellular fish scale (Table 2). Furthermore, histology results showed a synergistic effect of PRP with fish scale scaffolds, when the main goal is enhancing bone marrow regeneration despite negative results of some other studies (Table 2). Further studies with more laboratory animals and longer periods are required to assess the fish scale regenerative potential and follow the remodelling phase of bone healing.

\section{Acknowledgements}

We would like to gratefully acknowledge Dr Pejman Mortazavi for his contribution in histhopathological study, Dr Saeed Hosseini for his great contribution in in vitro preparation of materials and Dr Roozbeh Moridpour for his valuable support and fellowship.

\section{References}

Alsousou J, Thompson M, Hulley P, Noble A, Willett K 2009: The biology of platelet-rich plasma and its application in trauma and orthopaedic surgery: a review of the literature. J Bone Joint Surg Br 91: 987-996

Bansal MR, Bhagat SB, Shukla DD 2009: Bovine cancellous xenograft in the treatment of tibial plateau fractures in elderly patients. Int Orthop 33: 779-784

Buckwalter JA, Cooper RR 1987: Bone structure and function. Instr Course Lect 36: 2748

Campana V, Milano G, Pagano E, Barba M, Cicione C, Salonna G, Lattanzi W, Logroscino G 2014: Bone substitutes in orthopaedic surgery: from basic science to clinical practice. J Mater Sci Mater Med 25: 2445-2461

Chou CH, Chen YG, Lin CC, Lin SM, Yang KC, Chang SH 2014: Bioabsorbable fish scale for the internal fixation of fracture: a preliminary study. Tissue Eng Part A 20: 2493-2502

Civinini R, Macera A, Nistri L, Redl B, Innocenti M 2011: The use of autologous blood-derived growth factors in bone regeneration. Clin Cases Miner Bone Metab 8: 25-31

Develioglu H, Unver Saraydin S, Kartal U 2009: The bone healing effect of a xenograft in a rat calvarial defect model. Dent Mater J 28: 396400

Dimitriou R, Jones E, McGonagle D, Giannoudis P.V 2011: Bone regeneration: current concepts and future directions. BMC Med 9: 66

Dong J, Mo X, Li Y, Chen D 2012: Recent research progress of decellularization of native tissues. Sheng Wu Yi Xue Gong Cheng Xue Za Zhi 29: 100713

Durmuslar MC, Alpaslan C, Alpaslan G, Çakır M 2014: Clinical and radiographic evaluation of the efficacy of plateletrich plasma combined with hydroxyapatite bone graft substitutes in the treatment of intrabony defects in maxillofacial region. Acta Odontol Scand 72: 948-953

Gilbert TW, Sellaro TL, Badylak SF 2006: Decellularization of tissues and organs. Biomaterials 27: 3675-3683

Hakimi M, Jungbluth P, Sager M, Betsch M, Herten M, Becker J, Windolf J, Wild M 2010: Combined use of platelet rich plasma and autologous bone grafts in the treatment of long bone defects in minipigs. Injury 41: 71723

Hashimoto Y, Funamoto S, Kimura T, Nam K, Fujisato T, Kishida A 2011: Evaluation of decellularized bone using high-hydrostatic pressure. Biomaterials 32: 7060-7067

Hoyer B, Bernhardt A, Heinemann S, Stachel A, Meyer M, Gelinsky M 2012: Biomimetically mineralized salmon collagen scaffolds for application in bone tissue engineering. Biomacromolecules 13: 1059-1066

Kirshnan S, Sekar S, Katheem MF, Krishnakumar S, Sastry TP 2012: Fish scale collagen-a novel material for corneal tissue engineering. Artif Organs 36: 829-835

Korkmaz M, Ozturk H, Bulut O, Unsaldi T, Kaloglu C 2005: The effect of definitive continuous distraction employed with the Ilizarov type external fixation system on fracture healing: an experimental rabbit model. Acta Orthop Traumatol Turc 39: 247-257

Kovach TK, Dighe AS, Lobo PI, Cui Q 2015: Interactions between MSCs and immune cells: implications for bone healing. J Immunol Res 2015: 752510

Liao X, Lu S, Zhou Y, Winter C, Xu W, Li B, Wang Y 2011: Bone physiology, biomaterial and the effect of mechanical / physical microenvironment on MSC osteogenesis. Cell Mol Bioeng 4: 579-590

McMahon RE, Wang L, Skoracki R, Mathur AB 2013: Development of nanomaterials for bone repair and regeneration. J Biomed Mater Res B Appl Biomater 101: 387-397

Matsumoto R, Uemura T, Xu Z, Yamaguchi I, Ikoma T, Tanaka J 2015: Rapid oriented fibril formation of fish scale collagen fascilitates early osteoblastic differentiation of human mesenchymal stem cells. J Biomed Mater Res A 103: 2531-2539

Navarro M, Michiardi M, Castaño O, Planell A 2008: Biomaterials in orthopaedics. J R Soc Interface 5: $1137-1158$

Oryan A, Alidadi S, Moshiri A, Maffulli N 2014: Bone regenerative medicine: classic options, novel strategies, and future directions. J Orthop Surg Res 9: 18 
Pati F, Datta P, Adhikari B, Dhara S, Ghosh K, Das Mohapatra PK 2012: Collagen scaffolds derived from fresh water fish origin and their biocompatibility. J Biomed Mater Res A 100: 1068-1079

Roffi A, Filardo G, Kon E, Marcacci M 2013: Does PRP enhance bone integration with grafts, graft substitutes or implants? A systematic review. BMC Musculoskelet Disord 4: 330

Sarkar SK, Lee BT 2015: Hard tissue regeneration using bone substitutes: an update on innovations in materials. Korean J Intern Med 30: 279-293

Salamanna F, Veronesi F, Maglio M, Della Bella E, Sartori M, Fini M 2015: New and emerging strategies in platelet rich plasma application in musculoskeletal regenerative procedures: general overview on still open questions and outlook. Biomed Res Inj 2015: 846045

Schneppendahl J, Jungbluth P, Lögters TT, Sager M, Wild M, Hakimi M, Windolf J, Grassmann JP 2015: Treatment of a diaphyseal longbone defect with autologous bone grafts and platelet rich plasma in a rabbit model. Vet Comp Orthop Traumatol 28: 164-171

Sawkins MJ, Bowen W, Dhadda P, Markides H, Sidney LE, Taylor AJ, Rose FR, Badylak SF, Shakesheff KM, White LJ 2013: Hydrogels derived from demineralized and decellularized bone extracellular matrix. Acta Biomater 9: 7865-7873

Silva TH, Silva JM, Marques ALP, Domingues A, Bayon Y, Reis RL 2014: Marine origin collagens and It's potential applications. Mar Drugs 12: 5881-5901

To Minh Q, Doan Nguyen V, Tran Le, Bao H 2014: Decellularization of xenogenic bone grafts for potential use as tissue engineering scaffolds. IJLSDR 4: 38-46JJLSR

Thamamongood TA, Furuya R, Fukuba S, Nakamura M, Suzuki N, Hattori A 2012: Expression of osteoblastic and osteoclastic genes during spontaneous regeneration and autotransplantation of goldfish scale: a new tool to study intramembranous bone regeneration. Bone 50: 1240-1209

Wang Q, Gu Z, Jamal S, Detamore MS, Berkland C 2013: Hybrid hydroxyapatite nanoparticle colloidal gels are injectable fillers for bone tissue engineering. Tissue Eng Part A 9: 2586-2593

Yamada SH, Yamamoto K, Ikeda T, Yanagiguchi K, Hayashi Y 2014: Potency of fish collagen as a scaffold for regenerative medicine. Biomed Res Int 2014: 302932

Yamamoto K, Igawa K, Sugimoto K, Yoshizawa Y, Yanagiguchi K, Ikeda T, Yamada S, Hayashi Y 2014: Biological safety of fish (tilapia) collagen. Biomed Res Int 2014: 630757

Yilmaz S, Kabadayi C, Ipci SD, Caker G, Kuru B 2011: Treatment of intrabony periodontal defects with plateletrich plasma versus platelet-poor plasma combined with a bovine-derived xenograft: a controlled clinical trial. J Periodontol 82: 837-844

Yuan F, Wang L, Lin CC, Chou CH, Li L 2014: A cornea substitute derived from fish scale: 6 month follow up on rabbit model. J Ophthalmol 2014: 914542

Zigdon-Giladi H, Rudich U, Michaeli Geller G, Evron A 2015: Recent advances in bone regeneration using adult stem cells. World J Stem Cells 7: 630-640 
Plate VI

Mansouri N. et al.: The role of fish ... pp. 363-370
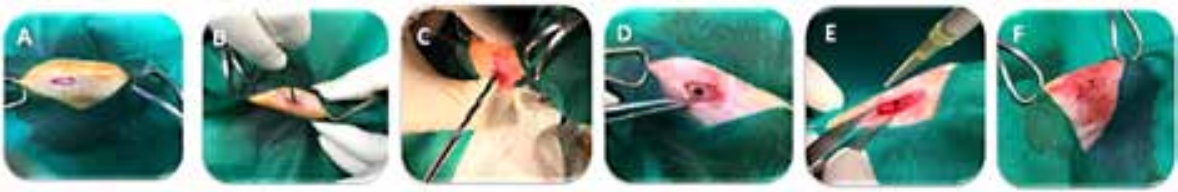

Fig. 1. Stages of surgical procedure. A: Skin incision. B. Periosteal elevation and bone exposure. C, D: Tibial drill hole in order to make a defect. E: Platelet rich plasma application. F: Subcutaneous tissue and skin suture.
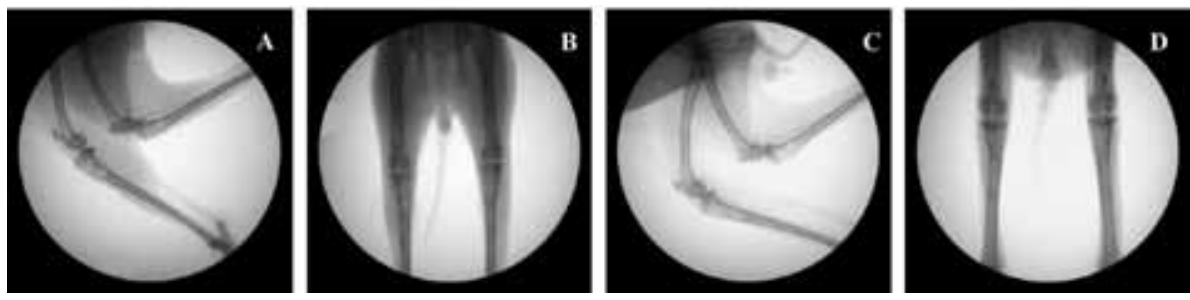

Fig. 2. Fluoroscopic view of hind limbs on day 0 (A, B) and on day 56 (C, D).

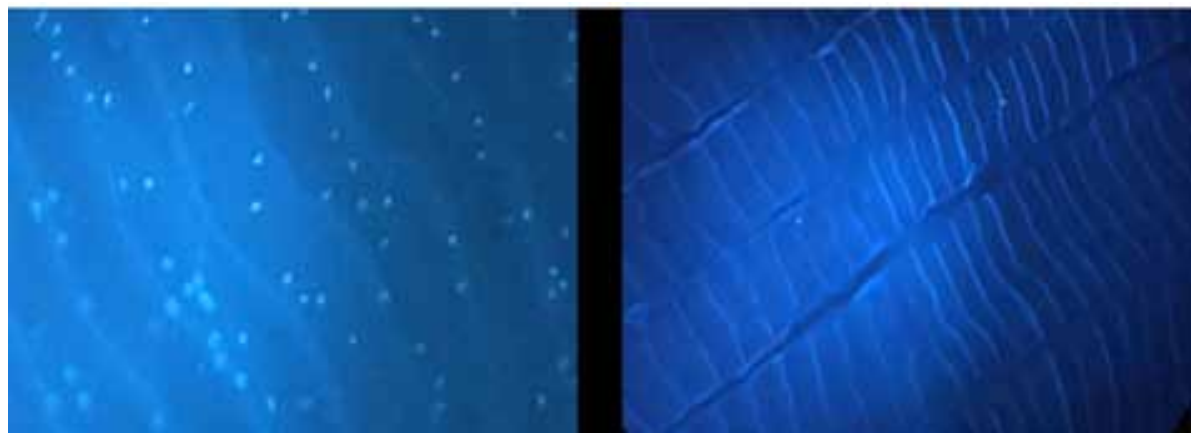

Fig. 3. Morphological and surface characteristics of fish scale before (A) and after (B) the decellularization process. 

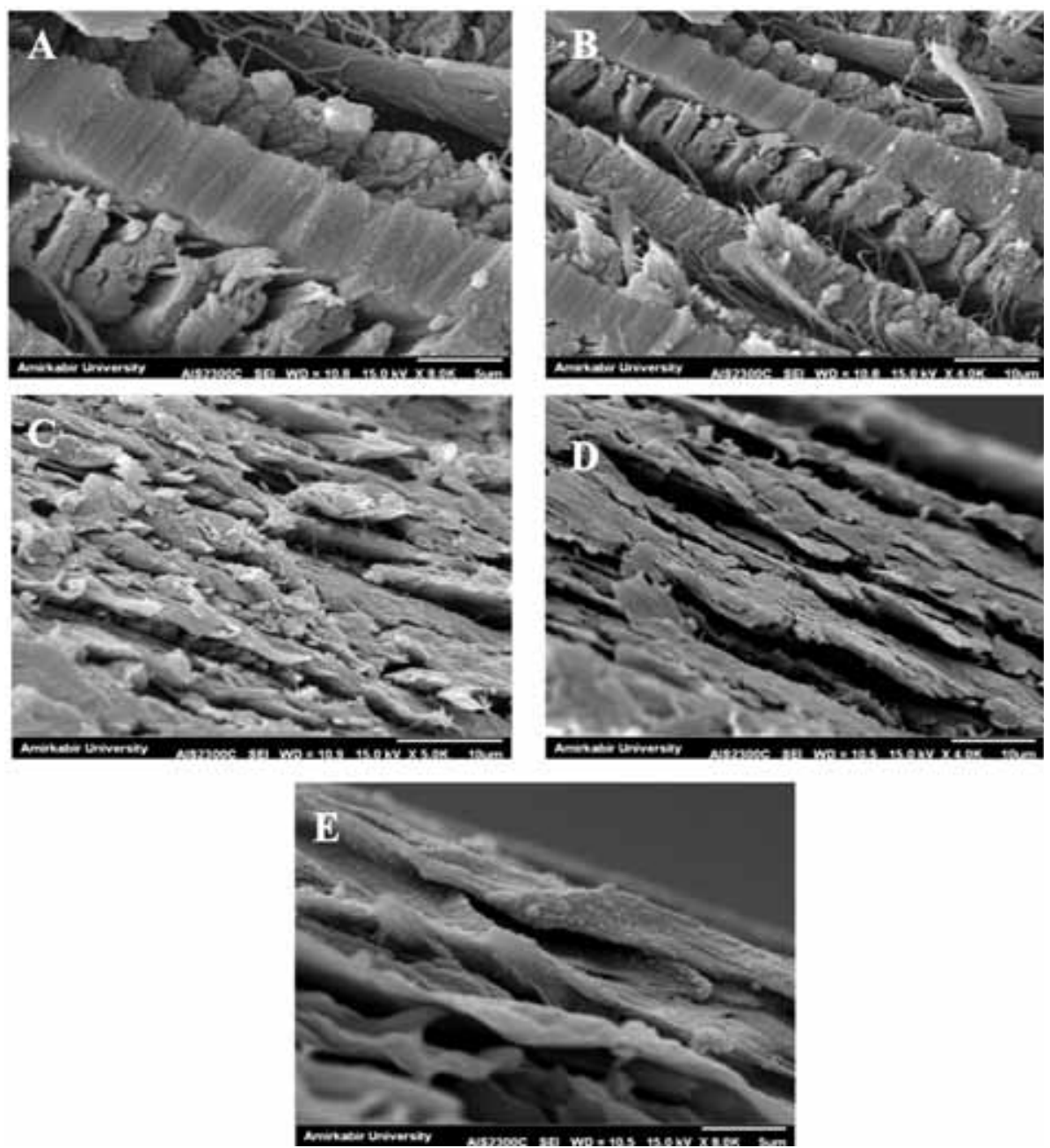

Fig. 4. Scanning electron microscopy image of fish scale before (A, B) and after (C, D, E) the decellularization process showing a layered collagen structure. 


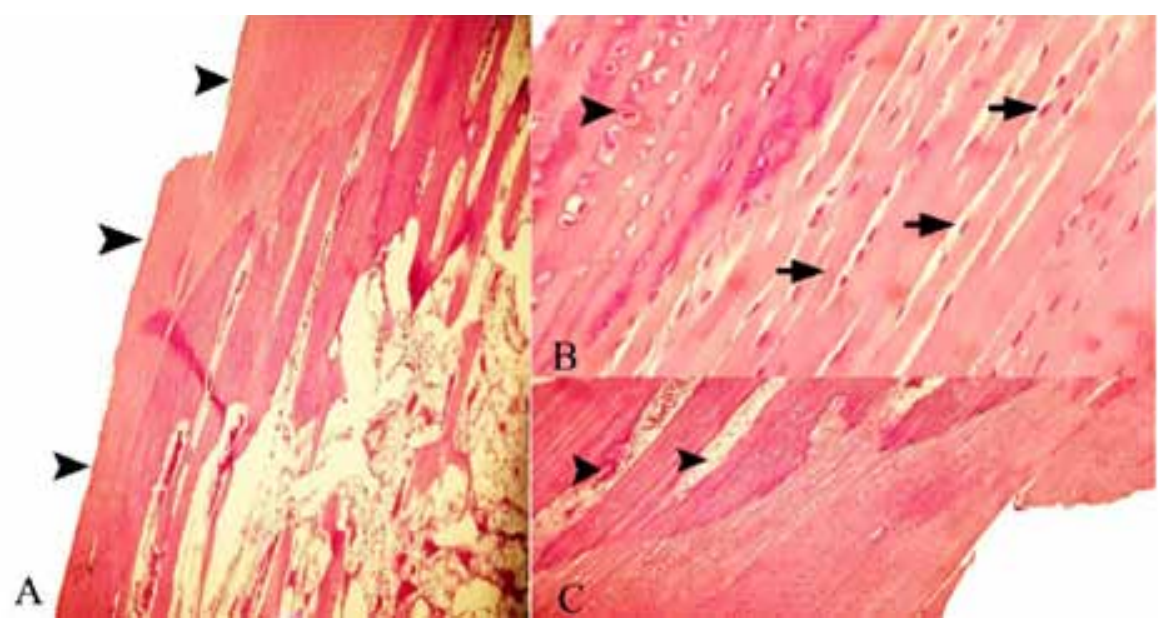

Fig. 5. The cortical defect site in the negative control group stained with haematoxyline and eosin on day 56. The defect site is covered by an extensive amount of fibrous connective tissue (arrowheads) and some cartilaginous callus $(\times 4)$. B: Collagen fibres and fibrocyte nuclei (arrows) in fibrous connective tissue, and chondrocytes in the cartilaginous callus (arrowhead) $(\times 40)$. C: Bone marrow cavities (arrowheads) and observable scarce cancellous tissue $(\times 10)$.

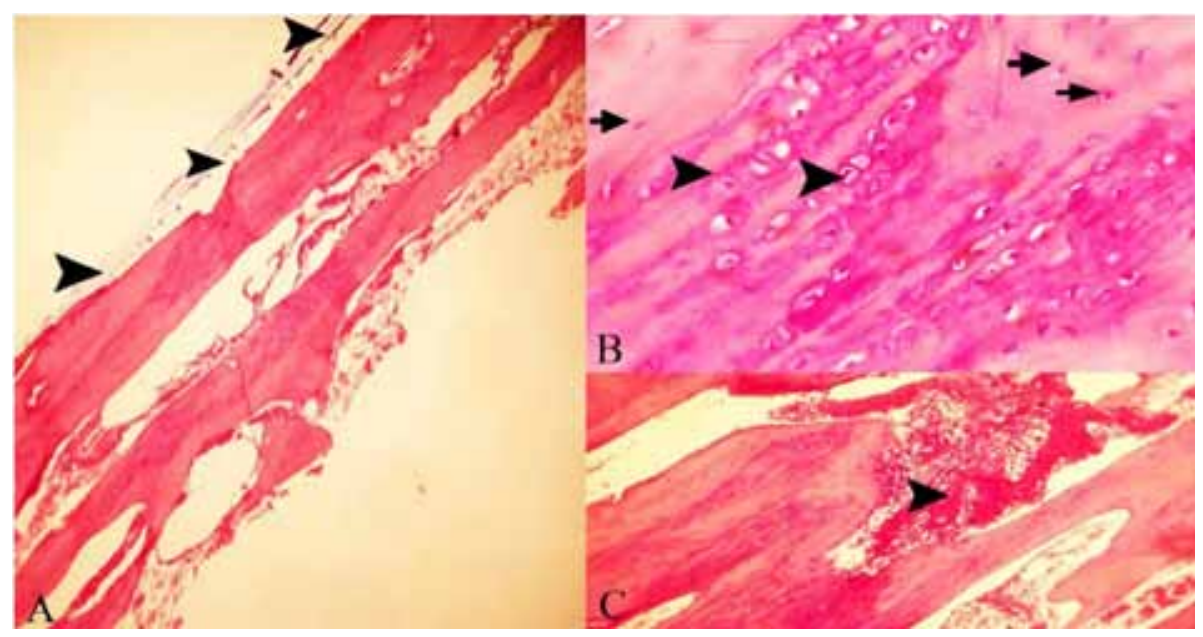

Fig. 6. The cortical defect site in group 1 stained with haematoxyline and eosin on day 56. A: Abundant osteocartilaginous callus is visible (arrowheads) $(\times 4)$. B: Chondrocytes (arrowheads) and osteocytes (arrows) at the healing site $(\times 40)$. C: Haematopoietic bone marrow has filled bone marrow cavities (arrowhead) $(\times 10)$. 


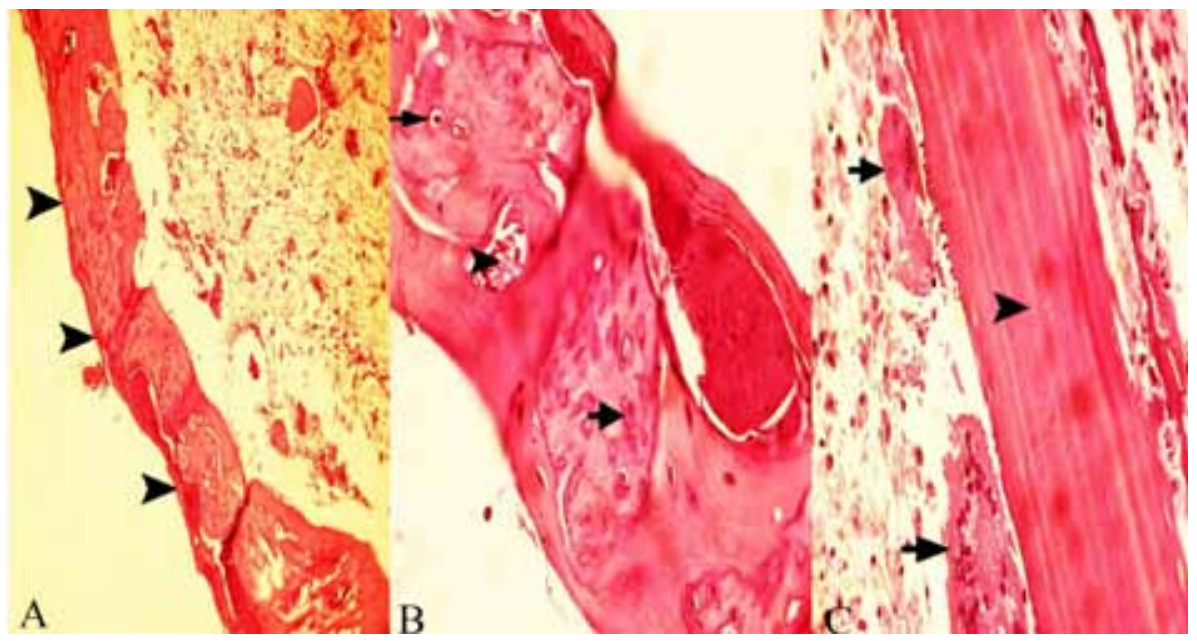

Fig. 7. The cortical defect site in group 2 stained with haematoxyline and eosin on day 56. A: Osseous tissue has covered the defect site $(\times 4)$. B: Bone marrow cavities (arrowhead) and hypertrophic osteocytes (arrows) are visible in the healing area $(\times 40)$. C: Scale residues surrounded by osteoclasts present in bone marrow cavities $(\times 40)$.

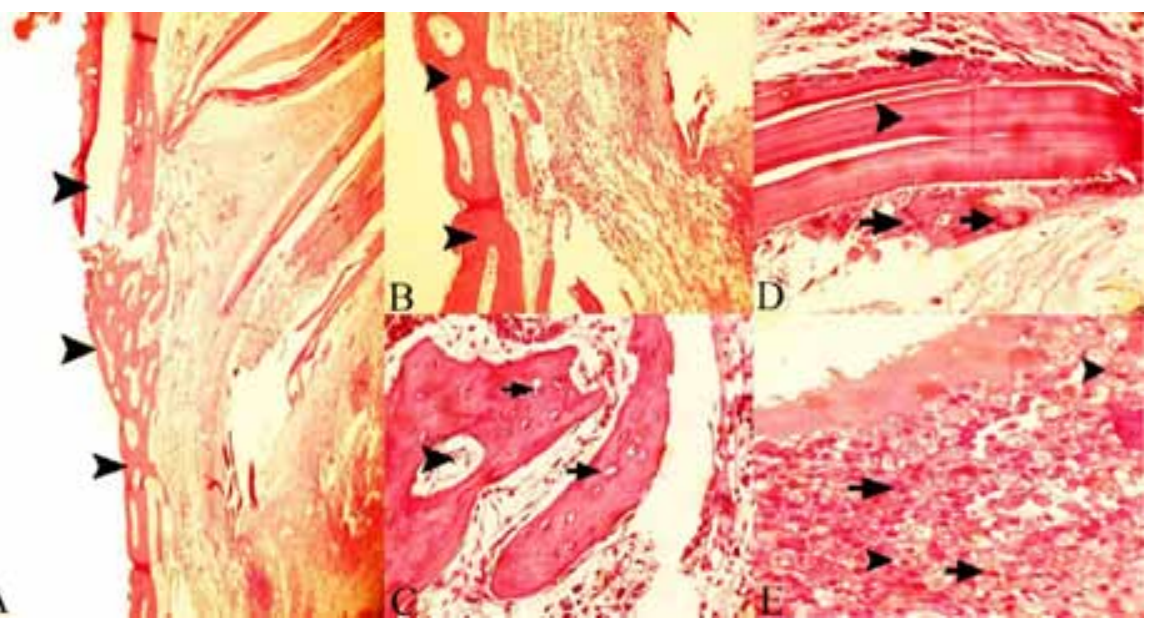

Fig. 8. The cortical defect site in group 3 stained with haematoxyline and eosin on day 56. A: Osseous tissue is observable at the defect site (arrowheads) $(\times 4)$. B: Osseous callus is present at the healing site (arrowheads) ( $\times 40)$. C: Hypertrophic osteocytes (arrows) and bone marrow cavities (arrowhead) are evident in the newly formed osseous callus $(\times 40)$. D: Fish scale residues are confined by osteoclasts (arrowhead) $(\times 40)$. E: Macrophages (arrowheads) and lymphocytes (arrows) have triggered an inflammatory response around fish scale residues $(\times 40)$. 


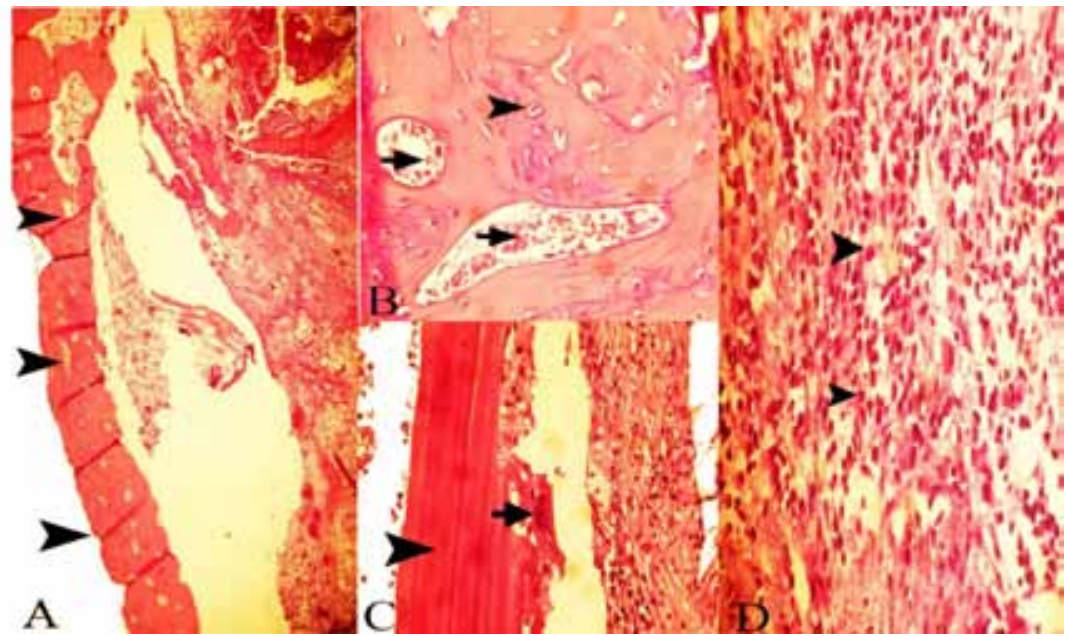

Fig. 9. The cortical defect site in group 4 stained with haematoxyline and eosin on day 56. A: Considerable osseous tissue is obvious at the defect site (arrowheads) $(\times 4)$. B: Newly formed osseous callus containing hypertrophic osteocytes (arrowhead) and blood-filled bone marrow cavities (arrows) are demonstrated $(\times 40)$. C: Osteoclasts (arrow) surrounding fish scale residues (arrowhead) are present $(\times 40)$. D: Severe inflammatory response with lymphocytes present around fish scale residues is evident $(\times 40)$.

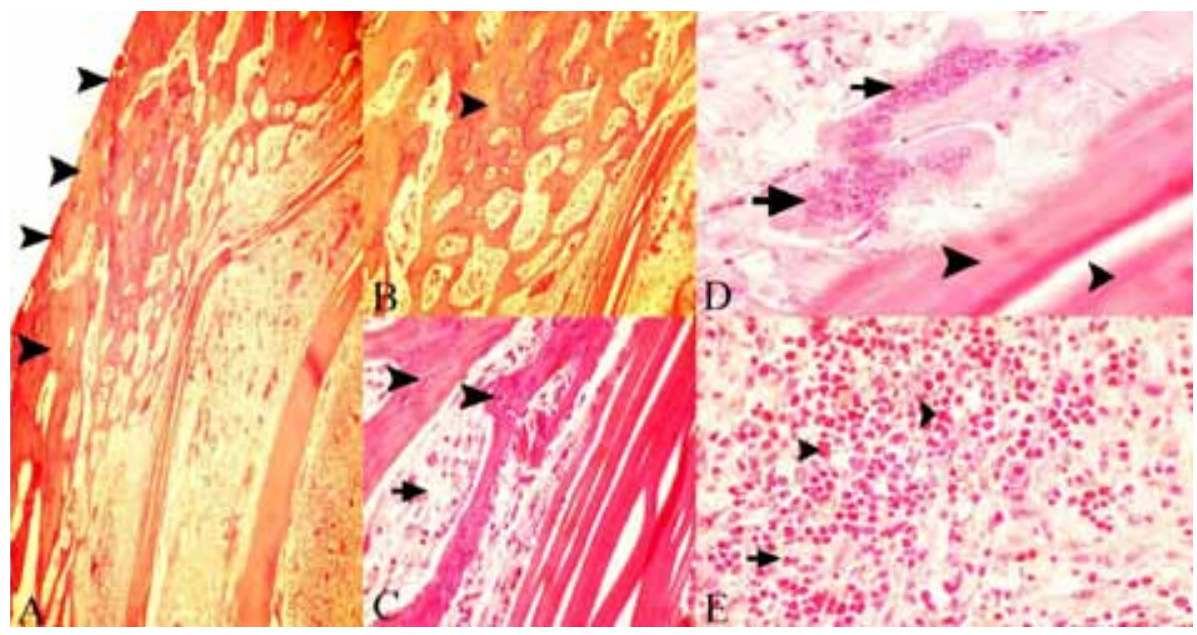

Fig. 10. The cortical defect site in group 5 stained with haematoxyline and eosin on day 56. A: Osseous tissue is present at the defect site (arrowheads) $(\times 4)$. B: Osseous callus is observable at the healing site (arrowhead) $(\times 40)$. C: Hypertrophic osteocytes (arrowheads) and mature bone marrow cavities (arrow) are visible $(\times 40)$. D: Fish scale residues in bone cavities (arrowheads) and several osteoclasts are visible (arrows) $(\times 40)$. E: Severe inflammatory response with presence of eosinophils (arrowheads), lymphocytes and plasma cells (arrow) $(\times 40)$. 